\title{
Radix Dipsaci total saponins stimulate MC3T3-E1 cell differentiation via the bone morphogenetic protein-2/MAPK/Smad-dependent Runx2 pathway
}

\author{
YIN-BO NIU $^{1}$, XIANG-HE KONG ${ }^{2}$, YU-HUA LI ${ }^{3}$, LI FAN ${ }^{1}$, YA-LEI PAN ${ }^{1}$, \\ CHEN-RUI LI ${ }^{1}$, XIANG-LONG WU ${ }^{1}$, TING-LI LU ${ }^{1}$ and QI-BING MEI ${ }^{1,3}$
}

\begin{abstract}
${ }^{1}$ Key Laboratory for Space Bioscience and Biotechnology, School of Life Sciences, Northwestern Polytechnical University, Xi'an, Shaanxi 710072; ${ }^{2}$ Graduate School of Chang'an University, Xi'an, Shaanxi 710064; ${ }^{3}$ Department of Pharmacology, School of Pharmacy, Fourth Military Medical University, Xi'an, Shaanxi 710032, P.R. China
\end{abstract}

Received March 24, 2014; Accepted December 9, 2014

DOI: $10.3892 / \mathrm{mmr} .2015 .3249$

\begin{abstract}
Radix Dipsaci total saponins (RTS) are primary active components of Radix Dipsaci, which is administered orally for the treatment of osteoporosis according to Chinese Medicine. RTS have also been shown to reduce the risk of bone fractures in rats. However, the detailed molecular mechanisms underlying their action remain elusive. In the present study, the ability of RTS to increase alkaline phosphatase activity, osteocalcin levels and the degree of mineralization was investigated in MC3T3-E1 mouse osteoblast precursor cells. In addition, the associated molecular mechanism was detected. The results revealed that RTS exerted an effect on osteoblastic maturation and differentiation. Induction of differentiation by RTS was associated with an increase in the expression levels of bone morphogenetic protein-2 (BMP-2), phosphorylated (P)-Smad1/5/8, P-ERK1/2, P-p38 and Runt-related transcription factor 2 (Runx2). Blocking BMP-2 expression with noggin significantly reduced the levels of osteoblastic differentiation and subsequently attenuated the expression levels of P-Smad1/5/8, P-ERK1/2, P-p38 and Runx2. This indicated that RTS induced osteoblastic differentiation through BMP-2/mitogen-activated protein kinase/Smad1/5/8-dependent Runx2 signaling pathways and that it may be a promising agent for enhancing bone formation.
\end{abstract}

\section{Introduction}

The survival of osteoblasts is one of the determinants of the development of osteoporosis. The drugs currently used in

\footnotetext{
Correspondence to: Professor Qi-Bing Mei or Professor Ting-Li $\mathrm{Lu}$, Key Laboratory for Space Bioscience and Biotechnology, School of Life Sciences, Northwestern Polytechnical University, 127 Youyi West Road, Xi'an, Shaanxi 710072, P.R. China

E-mail: qbmei@nwpu.edu.cn

E-mail: lutinglixinxin@nwpu.edu.cn
}

Key words: MC3T3-E1 cells, differentiation the treatment of osteoporosis are bone resorption inhibitors, including bisphosphonates, calcitonin and estrogen $(1,2)$. However, the positive effect of these drugs on the recovery of bone mass is moderate (1). Therefore, satisfactory anabolic agents are urgently required. Through increasing the proliferation of an osteoblastic lineage, or inducing differentiation and mineralization, these agents stimulate an increase in bone tissue and the prevention of bone destruction $(3,4)$.

As mediators in the cell signaling pathways associated with bone formation, bone morphogenetic proteins (BMPs) have an important role in the differentiation of osteoblasts (5-7). The Smad and mitogen-activated protein kinase (MAPK) pathways are essential components of BMP signaling during osteoblast differentiation (8-10). Among the members of the BMP subfamily, BMP-2 is able to induce bone formation and differentiation in vivo and in vitro $(11,12)$. BMP-2 activates ERK1/2, p38, c-Jun kinases and Smad1/5/8 proteins (13-16), and induces the expression of core binding factor (Cbfa)1/Runt-related transcription factor 2 (Runx2) (17,18), an important transcription factor in osteoblastic differentiation (19).

A previous study by our group demonstrated that RTS effectively inhibited osteoporosis in ovariectomized rats. Additionally, RTS has been shown to enhance MC3T3-E1 cell differentiation, potentially due to its role in increasing the expression levels of BMP-2 (20). However, the detailed molecular mechanisms of the osteogenic effects of RTS remain to be determined. In the present study, the effects of RTS on the osteogenic activities of MC3T3-E1 cells were investigated. In addition, in order to establish the potential mechanisms involved in the osteoprotective effects of RTS, the levels of BMP-2, Smad1/5/8, MAPKs and Runx2 were assayed, proteins which are associated with the osteogenesis signaling pathways.

\section{Materials and methods}

Cells and reagents. MC3T3-E1 cells were obtained from the American Type Culture Collection (Manassas, VA, USA) and maintained in $\alpha$-minimum essential medium ( $\alpha$-MEM; 
HyClone Laboratories, Inc., Logan, UT, USA) containing $10 \%$ fetal bovine serum (FBS; Hyclone Laboratories, Inc.) and $1 \%$ penicillin/streptomycin (Sigma-Aldrich, St. Louis, MO, USA) in a humidified atmosphere of $5 \% \mathrm{CO}_{2}$ and $95 \%$ air at $37^{\circ} \mathrm{C}$. Radix Dipsaci was purchased in a local Chinese Medicine store and was identified morphologically, histologically and chemically using standard Chinese Pharmacopoeia procedures (Chinese Pharmacopeia Commission, 2010). In brief, RTS was isolated and purified by refluxing in $60 \%$ ethanol and D101 macroporous resin, repectively. RTS was analyzed by colorimetric determination using asperosaponin VI (Push Bio-Technology Co. Ltd , Chengdu, China) as the standard and the content of RTS was $76.5 \%$ (23).

Alkaline phosphatase (ALP) activity assay. Osteoblasts were seeded at a density of $2 \times 10^{4}$ cells/well and cultured in 24-well plates with $\alpha$-MEM containing $10 \%$ FBS, L-ascorbic acid $(50 \mu \mathrm{g} / \mathrm{ml})$ and $\beta$-glycerophosphate $(10 \mathrm{mM})$ in the presence or absence of RTS $(30,100$ or $300 \mu \mathrm{g} / \mathrm{ml})$. The cells were washed twice with phosphate-buffered saline (PBS; Wuhan Boster Biological Technology, Inc., Wuhan, China), lysed with 0.2\% Triton X-100 (Tianjin Damao Chemical Reagent Factory, Tianjin, China) and the lysate was centrifuged at $14,000 \times \mathrm{g}$ for $5 \mathrm{~min}$. The supernatant was collected in order to measure the ALP activity and protein concentration using an ALP activity assay kit (Nanjing Jiancheng Bioengineering Institute, Nanjing, Chian) and a BCA protein assay kit (Beyotime, Shanghai, China), respectively (21).

Assessment of osteocalcin. Osteocalcin ELISA kits (Elabscience Biotechnology Co., Ltd, Wuhan, China) were used to detect osteocalcin levels. Cells were treated with RTS $(30,100$ or $300 \mu \mathrm{g} / \mathrm{ml})$ for eight days. The samples were placed in 96-well microtiter plates coated with mouse monoclonal detective antibodies for osteocalcin from the kit and incubated for $2 \mathrm{~h}$ at room temperature according to the manufacturer's instructions. Following removal of the unbound material using washing buffer, horseradish peroxidase (HP)-conjugated streptavidin was added to bind to the antibodies. HP catalyzes the conversion of the chromogenic tetramethylbenzidine to a colored solution, with the color intensity in proportion to the amount of protein present in the sample. The absorbance of each well was measured at a wavelength of $450 \mathrm{~nm}$ (Synergy ${ }^{\mathrm{TM}}$ HT Multi-Mode Microplate Reader; Bio-Tek Instruments, Inc., Winooski, VT, USA). Results are presented as the percentage change in the activity of the treated cells compared with that of the untreated control.

Mineralized matrix assay. Mineralization was determined via staining with Alizarin Red-S (Aldrich, Milwaukee, WI, USA). MC3T3-E1 cells were seeded into 12-well plates at a density of $2 \times 10^{5}$ cells/well. Following two days of incubation, cells were washed twice with PBS solution, treated with or without RTS and cultured in $\alpha$-MEM containing $10 \%$ FBS, L-ascorbic acid (50 $\mu \mathrm{g} / \mathrm{ml}$; Sigma-Aldrich) and $\beta$-glycerophosphate $(10 \mathrm{mM}$; Sigma-Aldrich). During this period, the medium was changed every three days. Following a 14-day incubation with or without drugs, the cells were fixed with $70 \%$ ethanol for $1 \mathrm{~h}$, washed three times with distilled water and then incubated with $40 \mathrm{mmol} / \mathrm{l}$ Alizarin Red-S (pH 4.2) for $10 \mathrm{~min}$ at $37^{\circ} \mathrm{C}$. Once stained, the cultures were washed three times with deionized water and then incubated with PBS for a further $15 \mathrm{~min}$. Images of the mineralized matrices were captured using a microscope (80i; Nikon, Tokyo, Japan). To quantify the matrix mineralization, Alizarin Red-S-stained cultures were incubated in $100 \mathrm{mmol} / \mathrm{l}$ cetylpyridinium chloride (Tianjin Damao Chemical Reagent Factory) for $1 \mathrm{~h}$ in order to solubilize and release calcium-bound Alizarin Red-S into the solution. The absorbance of the released Alizarin Red-S (Sigma-Aldrich) was measured at a wavelength of $570 \mathrm{~nm}$ (Synergy $^{\mathrm{TM}}$ HT Multi-Mode Microplate Reader; Bio-Tek) (24).

Western blot analysis. To detect protein expression following RTS treatment, MC3T3-E1 cells were lysed and the cell lysates were harvested and maintained on ice for $30 \mathrm{~min}$. Once the soluble fractions of nuclear and cytoplasmic proteins were obtained they were used for western blotting. Equal amounts of protein were subjected to $15 \%$ SDS-PAGE (Wuhan Boster Biological Technology, Inc.). The proteins were transferred to nitrocellulose membranes (Pall, Port Washington, NY, USA) using transfer buffer (50 mM Tris, $190 \mathrm{mM}$ glycin and 10\% methanol; Tianjin Damao Chemical Reagent Factory) at $50 \mathrm{~V}$ for $2.5 \mathrm{~h}$. The membranes were incubated with blocking buffer containing $0.05 \%$ Tween-20 (Tianjin Damao Chemical Reagent Factory) and 5\% non-fat milk for $12 \mathrm{~h}$ at $4^{\circ} \mathrm{C}$. Following washing three times with PBS, the blot was incubated with primary antibodies (rabbit polyclonal anti-BMP-2, 1:200; rabbit polyclonal anti-Smad1/5/8, 1:200; rabbit polyclonal anti-phosphorylated (P)-Smad1/5/8, 1:200; rabbit polyclonal anti-p38, 1:200; rabbit polyclonal anti-P-p38, 1:200; rabbit polyclonal anti-ERK1/2, 1:200; rabbit polyclonal anti-P-ERK1/2, 1:200; rabbit polyclonal anti-JNK, 1:200; rabbit polyclonal anti-P-JNK, 1:200; rabbit polyclonal anti-Runx2, 1:200; rabbit polyclonal anti-histone, 1:500; and mouse monoclonal anti- $\beta$-actin, 1:1,000; Santa Cruz Biotechnology, Inc., Dallas, TX, USA) for $12 \mathrm{~h}$ at $4^{\circ} \mathrm{C}$. Subsequently, the membranes were washed three times for 10 min with tris-buffered saline (TBS) buffer containing $0.05 \%$ Tween-20 and subsequently incubated with anti-rabbit or anti-mouse immunoglobulin $\mathrm{G}$ secondary antibodies (1:5,000 dilution) for $1 \mathrm{~h}$ at room temperature. The membranes were washed three times for $10 \mathrm{~min}$ with Tris-buffered saline (Tianjin Damao Chemical Reagent Factory) and once for 10 min with PBS. Following reaction and coloration using enhanced chemiluminescence (Millipore, Billerica, MA, USA), the relative values for the absorbance of the bands and the absorbance of $\beta$-actin or histone were compared.

Statistical analysis. Data were analyzed using a one-way analysis of variance test to compare the different groups. Results are presented as the mean \pm standard deviation (SD). Statistical analyses were performed using SPSS 16.0 software (SPSS, Inc., Chicago, IL, USA). P $<0.05$ was considered to indicate a statistically significant difference.

\section{Results}

RTS enhances the differentiation of MC3T3-E1 cells. As shown in Fig. 1A, RTS treatment increased the level of ALP 
A

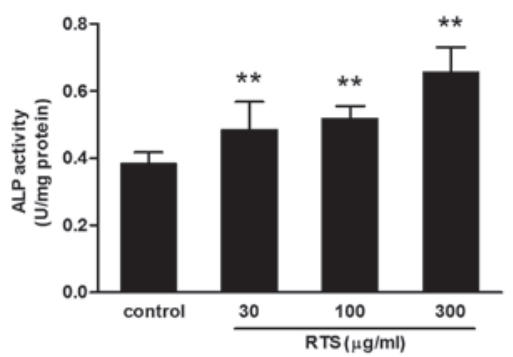

C



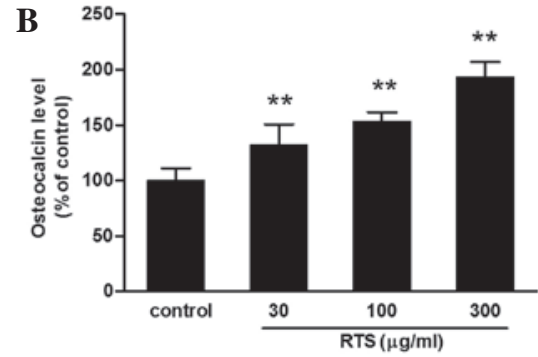



Figure 1. Enhancement of MC3T3-E1 cell differentiation by RTS. RTS increased (A) ALP activity and (B) osteocalcin production. Noggin inhibited the upregulation of (C) ALP and (D) osteocalcin production by RTS. ALP activity was assessed using a commercial ALP kit. The amount of osteocalcin in the culture medium was assessed using osteocalcin ELISA kits. Values are presented as the mean \pm standard deviation $(\mathrm{n}=6)$. $^{* *} \mathrm{P}<0.01 \mathrm{vs}$. control. RTS, Radix Dipsaci total saponins; ALP, alkaline phosphatase.
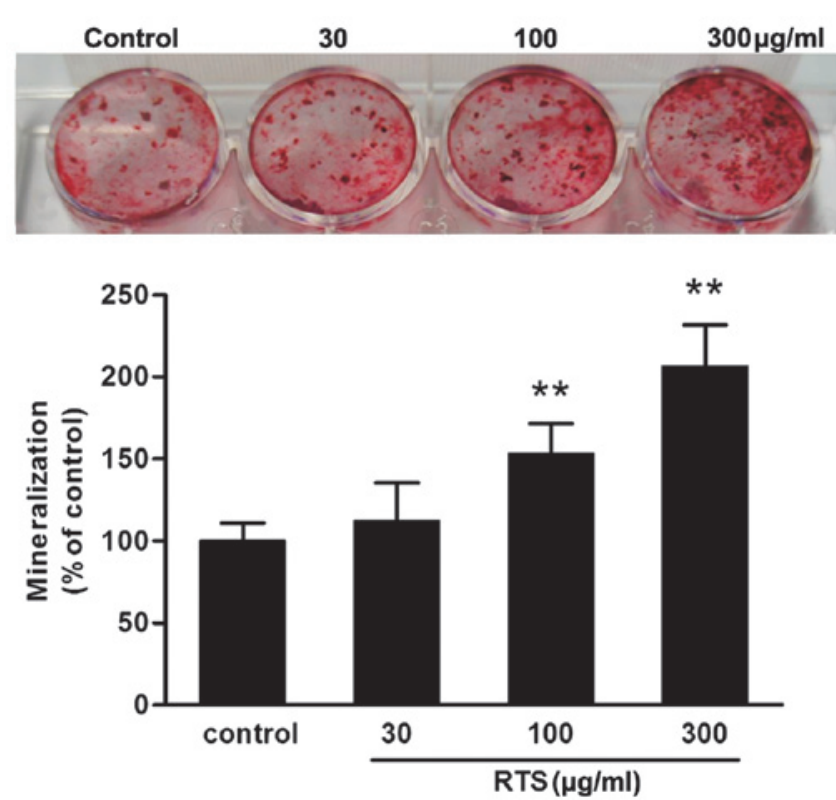

Figure 2. RTS increased the mineralization of MC3T3-E1 cells. The mineralized nodule formation was assessed by Alizarin Red-S staining. The bound stain was washed with a solution of $100 \mathrm{mmol} / 1$ cetylpyridinium chloride and quantified using a Bio-Rad ELISA reader. Values are presented as the mean \pm standard deviation $(n=3)$. ${ }^{* *} \mathrm{P}<0.01$ vs. control. RTS, Radix Dipsaci total saponins.

activity in a concentration-dependent manner. Additionally, the expression levels of osteocalcin protein were increased by RTS in a concentration-dependent manner in following 7 days of treatment (Fig. 1B); the levels in the 30, 100 and $300 \mu \mathrm{g} / \mathrm{ml}$ RTS-treated groups were significantly higher than those in the controls $(\mathrm{P}<0.01)$.

RTS promotes mineralization of MC3T3-E1 cells. The calcified nodules stained positive with Alizarin Red-S (Fig. 2).
The 100 and $300 \mu \mathrm{g} / \mathrm{ml}$ RTS-treated groups had the highest number of mineralized nodules $(\mathrm{P}<0.01$ compared with the control group).

RTS induces the differentiation of MC3T3-E1 cells via the BMP-2 pathway. As shown in Fig. 3A, BMP-2 protein levels were markedly increased by RTS treatment in a concentration-dependent manner. MC3T3-E1 cells were pretreated with noggin protein $(100 \mathrm{ng} / \mathrm{ml})$ for $2 \mathrm{~h}$, then $300 \mu \mathrm{g} / \mathrm{ml}$ RTS was added for $24 \mathrm{~h}$. RTS-induced BMP-2 protein expression was diminished by the concurrent treatment with noggin (Fig. 3B). In addition, the RTS-induced ALP activity and osteocalcin protein levels were significantly diminished in cells treated concurrently with noggin compared with those in untreated cells (Fig. 1C and D). Therefore, RTS-mediated cell differentiation may proceed via a BMP-2-dependent pathway.

BMP-2 is involved in the activation of Smad1/5/8, ERK and 338 in RTS-treated cells. Binding of BMP-2 to the BMP receptor activates MAPKs or SMADs via phosphorylation $(15,16)$. RTS treatment significantly increased the expression of P-Smad1/5/8, P-p38 and P-ERK1/2 (Fig. 3A). The activation of Smad1/5/8, p38 and ERK1/2 was blocked in MC3T3-E1 cells pretreated with $100 \mathrm{ng} / \mathrm{ml}$ noggin protein for $2 \mathrm{~h}$ and then co-treated with $300 \mu \mathrm{g} / \mathrm{ml}$ RTS for $24 \mathrm{~h}$ (Fig. 3B).

$B M P-2$ is required to increase expression of Runx2 in RTS-treated MC3T3-E1 cells. Runx 2 is a vital transcription factor required for osteoblast differentiation (19). Following treatment with RTS for $24 \mathrm{~h}$, the expression levels of Runx2 protein in cells were markedly increased (Fig. 4A). When cells were incubated with RTS in the presence of noggin, the stimulatory effect of RTS on the expression of Runx 2 protein were markedly reduced (Fig. 4B). These results indicated that RTS activated osteogenic differentiation through the BMP-2/MAPK/Smad-dependent Runx 2 signaling pathway. 


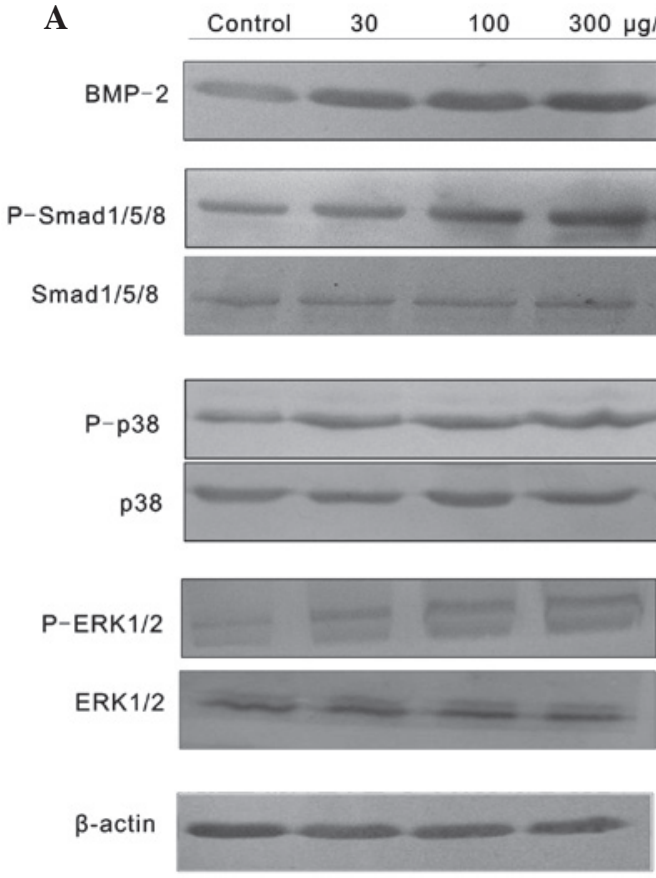

B
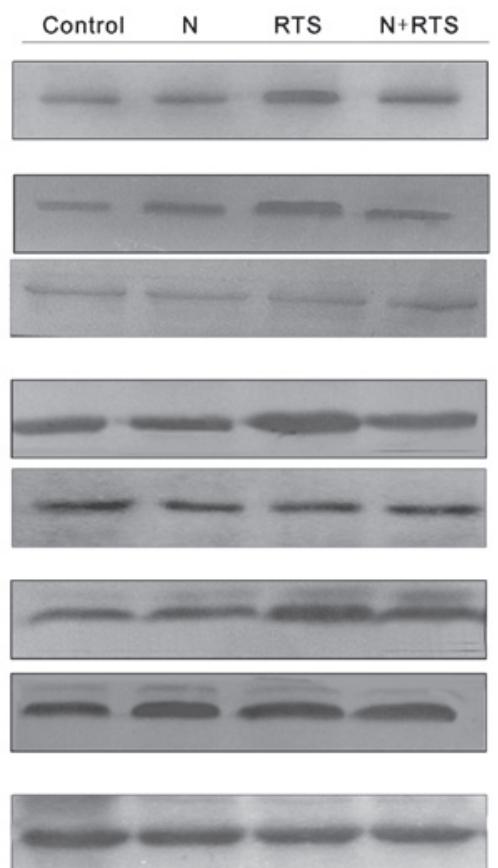

Figure 3. Western blots showing the protein levels of BMP-2, Smad1/5/8, p38 and ERK1/2 in MC3T3-E1 cells. (A) Protein in cells treated with RTS; (B) Noggin inhibited the induction of BMP-2, P-Smad1/5/8, P-p38 and P-ERK1/2 by RTS. Blots are representatives of three independent experiments. N, noggin; RTS, Radix Dipsaci total saponins; P, phosphorylated protein; BMP, bone morphogenetic protein.

A

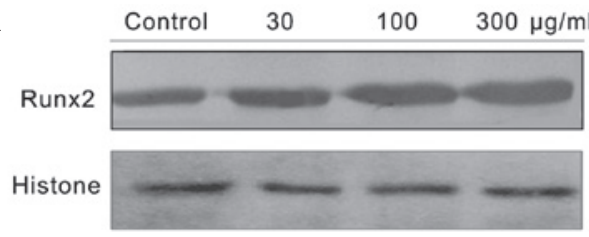

B

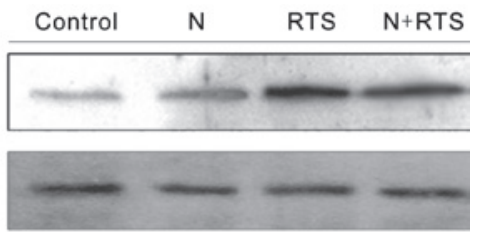

Figure 4. Western blots showing the protein levels of Runx2 in MC3T3-E1 cells. (A) Runx2 levels following treatment with RTS; (B) Noggin inhibited the induction of Runx2 by RTS. Blots are representatives of three independent experiments. N, noggin; RTS, Radix Dipsaci total saponins.

\section{Discussion}

The anti-osteoporosis effect of RTS in vivo was systemically evaluated in a previous study by our group (20). In the present study, the effects of RTS on the osteogenic activities in MC3T3-E1 cells and the potential mechanisms of action were evaluated. Treatment of MC3T3-E1 cells with RTS not only raised the activity of ALP (a marker of maturation) but also increased the levels of osteocalcin proteins (late stage markers of differentiation). This indicated that RTS may affect cell differentiation processes from the early to terminal stages. In addition, RTS increased the mineralization (a marker of bone formation) of MC3T3-E1 cells.

BMPs have potent osteogenic effects and control osteoblast differentiation during osteogenesis. BMP-2, one of the BMP subfamilies, promotes differentiation through enhanced intracellular ALP activity as well as osteocalcin and collagen protein synthesis (23). The effect of BMPs is mediated by heterotetrameric serine/threonine kinase receptors and the downstream transcription factors Smad1/5/8. Upon phosphorylation by type I receptors, Smad1/5/8 form complexes with Smad4, translocate to the nucleus and regulate the transcription of target genes associated with differentia- tion (13). Several natural or chemical compounds, including daidzein, osthole and syringetin, have been reported to induce osteoblast differentiation by induction of BMP and/or SMAD signaling (24-26). The results of the present study showed that the expression levels of BMP-2 were enhanced and the phosphorylation of SMAD1/5/8 was significantly increased in RTS-treated MC3T3-E1 cells. RTS-mediated SMAD1/5/8 activation was blocked by the BMP antagonist noggin and cell differentiation was attenuated in MC3T3-E1 cells. Hence, the BMP-2 signaling system has an important role in RTS-mediated cell maturation and differentiation in MC3T3-E1 cells.

In addition to Smad activation, BMP-2 can activate Smad-independent pathways, for example the MAPK signaling pathway. BMP-2 can stimulate two MAPKs: ERK and p38. The activation of p38 and ERK is essential in the BMP-2-induced upregulation of AP, type I collagen, osteocalcin and osteopontin (27-29). The results of the present study showed an increase in p38 and ERK activity in RTS-treated cells. This suggested that the activation of p38 and ERK may have an important role in increasing BMP-2 levels and the cell differentiation in MC3T3-E1 cells stimulated by RTS. The increase in the expression of Runx2 protein by RTS is prevented by the BMP inhibitor noggin, 
which demonstrates an involvement of the BMP-2 pathway in the stimulatory effect of RTS on Runx2.

In conclusion, the present study clearly demonstrated that RTS stimulates osteoblast differentiation at various stages in MC3T3-E1 cells. The effect of RTS on cell maturation and differentiation is strongly associated with the BMP-2/MAPK/Smad1/5/8-dependent Runx2 signaling pathway. This suggests that RTS may be beneficial in stimulating osteoblastic activity resulting in bone formation.

\section{Acknowledgements}

This study was financially supported by grants from the National Natural Science Foundation of China (no. 81202457), the China Postdoctoral Science Foundation (no. 2012T50822) and the Fundamental Research Foundation of Northwestern Polytechnical University (no. 3102014JKY15013).

\section{References}

1. Rodan GA and Martin TJ: Therapeutic approaches to bone diseases. Science 289: 1508-1514, 2000.

2. Schelonka EP and Usher A: Ipriflavone and osteoporosis. JAMA 286: 1836-1837, 2001.

3. Berg C, Neumeyer K and Kirkpatrick P: Teriparatide. Nat Rev Drug Discov 2: 257-258, 2003.

4. Lane NE and Kelman A: A review of anabolic therapies for osteoporosis. Arthritis Res Ther 5: 214-222, 2003.

5. Reddi AH: Bone and cartilage differentiation. Curr Opin Genet Dev 4: 737-744, 1994.

6. Sakou T, Onishi T, Yamamoto T, Nagamine T, Sampath T and Ten Dijke P: Localization of Smads, the TGF-beta family intracellular signaling components during endochondral ossification. J Bone Miner Res 14: 1145-1152, 1999.

7. Tang $\mathrm{CH}$, Yang RS, Huang TH, Liu $\mathrm{SH}$ and $\mathrm{Fu}$ WM: Enhancement of fibronectin fibrillogenesis and bone formation by basic fibroblast growth factor via protein kinase C-dependent pathway in rat osteoblasts. Mol Pharmacol 66: 440-449, 2004.

8. Gallea S, Lallemand F, Atfi A, et al: Activation of mitogen-activated protein kinase cascades is involved in regulation of bone morphogenetic protein-2-induced osteoblast differentiation in pluripotent C2C12 cells. Bone 28: 491-498, 2001.

9. Fujii M, Takeda K, Imamura T, et al: Roles of bone morphogenetic protein type I receptors and Smad proteins in osteoblast and chondroblast differentiation. Mol Biol Cell 10: 3801-3813, 1999.

10. Yamamoto N, Akiyama S, Katagiri T, Namiki M, Kurokawa T and Suda T: Smad1 and smad5 act downstream of intracellular signalings of BMP-2 that inhibits myogenic differentiation and induces osteoblast differentiation in $\mathrm{C} 2 \mathrm{C} 12$ myoblasts. Biochem Biophys Res Commun 238: 574-580, 1997.

11. Jia TL, Wang HZ, Xie LP, Wang XY and Zhang RQ: Daidzein enhances osteoblast growth that may be mediated by increased bone morphogenetic protein (BMP) production. Biochem Pharmacol 65: 709-715, 2003.

12. Takuwa Y, Ohse C, Wang EA, Wozney JM and Yamashita K: Bone morphogenetic protein-2 stimulates alkaline phosphatase activity and collagen synthesis in cultured osteoblastic cells MC3T3-E1. Biochem Biophys Res Commun 174: 96-101, 1991.
13. Nohe A, Keating E, Knaus P and Petersen NO: Signal transduction of bone morphogenetic protein receptors. Cell Signal 16: 291-299, 2004

14. Sykaras N and Opperman LA: Bone morphogenetic proteins (BMPs): how do they function and what can they offer the clinician? J Oral Sci 45: 57-73, 2003.

15. Gallea S, Lallemand F, Atfi A, et al: Activation of mitogen-activated protein kinase cascades is involved in regulation of bone morphogenetic protein-2-induced osteoblast differentiation in pluripotent $\mathrm{C} 2 \mathrm{C} 12$ cells. Bone 28 : 491-498, 2001.

16. Guicheux J, Lemonnier J, Ghayor C, Suzuki A, Palmer G and Caverzasio J: Activation of p38 mitogen-activated protein kinase and c-Jun- $\mathrm{NH}_{2}$-terminal kinase by BMP-2 and their implication in the stimulation of osteoblastic cell differentiation. J Bone Miner Res 18: 2060-2068, 2003.

17. Hsieh TP, Sheu SY, Sun JS, Chen MH and Liu MH: Icariin isolated from Epimedium pubescens regulates osteoblasts anabolism through BMP-2, SMAD4, and Cbfa1 expression. Phytomedicine 17: 414-423, 2010.

18. Lee KS, Hong SH and Bae SC: Both the Smad and p38 MAPK pathways play a crucial role in Runx2 expression following induction by transforming growth factor-beta and bone morphogenetic protein. Oncogene 21: 7156-7163, 2002.

19. Sakai S, Tamura M, Mishima H, Kojima H and Uemura T: Bone regeneration induced by adenoviral vectors carrying til-1/Cbfa1 genes implanted with biodegradable porous materials in animal models of osteonecrosis of the femoral head. J Tissue Eng Regen Med 2: 164-167, 2008

20. Niu YB, Li YH, Kong XH, et al: The beneficial effect of Radix Dipsaci total saponins on bone metabolism in vitro and in vivo and the possible mechanisms of action. Osteoporos Int 23: 2649-60, 2012.

21. Wong RW, Rabie AB and Hägg EU: The effect of crude extract from Radix Dipsaci on bone in mice. Phytother Res 21: 596-598, 2007.

22. Yang RS, Lin WL, Chen YZ, et al: Regulation by ultrasound treatment on the integrin expression and differentiation of osteoblasts. Bone 36: 276-283, 2005.

23. Sumanasinghe RD, Bernacki SH and Loboa EG: Osteogenic differentiation of human mesenchymal stem cells in collagen matrices: effect of uniaxial cyclic tensile strain on bone morphogenetic protein (BMP-2) mRNA expression. Tissue Eng 12: 3459-3465, 2006.

24. Jia TL, Wang HZ, Xie LP, Wang XY and Zhang RQ: Daidzein enhances osteoblast growth that may be mediated by increased bone morphogenetic protein (BMP) production. Biochem Pharmacol 65: 709-715, 2003.

25. Kuo PL, Hsu YL, Chang CH and Chang JK: Osthole-mediated cell differentiation through bone morphogenetic protein-2/p38 and extracellular signal-regulated kinase $1 / 2$ pathway in human osteoblast cells. J Pharmacol Exp Ther 314: 1290-1299, 2005.

26. Hsu YL, Liang HL, Hung CH and Kuo PL: Syringetin, a flavonoid derivative in grape and wine, induces human osteoblast differentiation through bone morphogenetic protein-2/extracellular signal-regulated kinase 1/2 pathway. Mol Nutr Food Res 53: 1452-61, 2009.

27. Attisano L and Wrana JL: Signal transduction by the TGF-beta superfamily. Science 296: 1646-1647, 2002.

28. Lai CF and Cheng SL: Signal transductions induced by bone morphogenetic protein-2 and transforming growth factor-beta in normal human osteoblastic cells. J Biol Chem 277: 15514-15522, 2002.

29. Weston CR, Lambright DG and Davis RJ: Signal transduction. MAP kinase signaling specificity. Science 296: 2345-2347, 2002. 